\title{
Autologous CD8+ Melanoma Specific T Cells
}

National Cancer Institute

\section{Source}

National Cancer Institute. Autologous CD8+ Melanoma Specific T Cells. NCI Thesaurus. Code C116072.

Autologous CD8 T-lymphocytes against melanoma-associated antigens, with potential immunomodulating and antineoplastic activities. Following leukapheresis and the ex vivo expansion of cytotoxic T-lymphocytes, the autologous CD8+ melanoma specific T-cells are re-introduced into the melanoma patient. These cytotoxic T-cells recognize and kill the patient's own melanoma cells. 